\title{
3-Equitable Prime Cordial Labeling of Graphs
}

\author{
S. Murugesan \\ Department of Mathematics \\ C. B. M. College, Kovaipudur, \\ Coimbatore - 641042 , \\ Tamil Nadu, INDIA. \\ smurugesancbmc@gmail.com
}

\author{
D. Jayaraman \\ Department of Mathematics \\ C. B. M. College, Kovaipudur, \\ Coimbatore - 641 042, Tamil Nadu, INDIA. \\ arunsrgt.2008@gmail.com
}

\author{
J. Shiama \\ Department of Mathematics, \\ C. M. S. College of Science and Commerce, \\ Coimbatore- 641 049, Tamil Nadu, INDIA. \\ jshiama@gmail.com
}

\begin{abstract}
A 3-equitable prime cordial labeling of a graph $G$ with vertex set $V$ is a bijection $f$ from $V$ to $\{1,2, \ldots,|V|\}$ such that if an edge $u v$ is assigned the label 1 if $\operatorname{gcd}(f(u), f(v))=1$ and $\operatorname{gcd}(f(u)+$ $f(v), f(u)-f(v))=1$, the label 2 if $g c d(f(u), f(v))=1$ and $\operatorname{gcd}(f(u)+f(v), f(u)-f(v))=2$ and 0 otherwise, then the number of edges labeled with $i$ and the number of edges labeled with $j$ differ by atmost 1 for $0 \leq i, j \leq 2$. If a graph has a 3 -equitable prime cordial labeling, then it is called a 3 -equitable prime cordial graph. In this paper, we investigate the 3 -equitable prime cordial labeling behaviour of paths, cycles, star graphs and complete graphs.
\end{abstract}

\section{Keywords:}

3-equitable prime cordial labeling, 3-equitable prime cordial graph

\section{INTRODUCTION}

The graphs considered here are finite, connected, undirected and simple. The vertex set and edge set of a graph $G$ are denoted by $V(G)$ and $E(G)$ respectively. For various graph theoretic notations and terminology we follow Harary [1] and for number theory we follow Burton [2]. We will give the brief summary of definitions which are useful for the present investigations.

DEFINITION 1. If the vertices of the graph are assigned values subject to certain conditions it is known as graph labeling.

Most interesting graph labeling problems have three important characteristics.

1. A set of numbers from which vertex labels are chosen.

2. A rule that assigns a value to each edge.

3 . A condition that these values must satisfy.

For detailed survey on graph labeling one can refer Gallian [3]. Vast amount of literature is available on different types of graph labeling. According to Beineke and Hegde [4] graph labeling serves as a frontier between number theory and structure of graphs.

Labeled graphs have variety of applications in coding theory, particularly for missile guidance codes, design of good radar type codes and convolution codes with optimal autocorrelation properties. Labeled graph plays vital role in the study of X-ray crystallography, communication network and to determine optimal circuit layouts. A detailed study of variety of applications of graph labeling is given by Bloom and Golomb [5].
The present work is to aimed to discuss one such labeling known as 3-equitable prime cordial labeling.

DEFINITION 2. Let $G=(V(G), E(G))$ be a graph. A mapping $f: V(G) \rightarrow\{0,1\}$ is called binary vertex labeling of $G$ and $f(v)$ is called the label of the vertex $v$ of $G$ under $f$.

For an edge $e=u v$, the induced edge labeling $f^{*}: E(G) \rightarrow\{0,1\}$ is given by $f^{*}(e)=|f(u)-f(v)|$. Let $v_{f}(0), v_{f}(1)$ be the number of vertices of $G$ having labels 0 and 1 respectively under $f$ and $e_{f}(0), e_{f}(1)$ be the number of edges having labels 0 and 1 respectively under $f^{*}$.

DEFINITION 3. A binary vertex labeling of a graph $G$ is called a cordial labeling if $\left|v_{f}(0)-v_{f}(1)\right| \leq 1$ and $\mid e_{f}(0)-$ $e_{f}(1) \mid \leq 1$. A graph $G$ is cordial if it admits cordial labeling.

The concept of cordial labeling was introduced by Cahit [6].

Many researchers have studied cordiality of graphs. Cahit [6] proved that tree is cordial and $K_{n}$ is cordial if and only if $n \leq 3$. Vaidya et al. [7] have also discussed the cordiality of various graphs.

Definition 4. Let $G=(V, E)$ be a graph. A mapping $f$ : $V(G) \rightarrow\{0,1,2\}$ is called ternary vertex labeling of $G$ and $f(v)$ is called the label of the vertex $v$ of $G$ under $f$.

For an edge $e=u v$, the induced edge labeling $f^{*}: E(G) \rightarrow\{0,1,2\}$ is given by $f^{*}(e)=|f(u)-f(v)|$. Let $v_{f}(0), v_{f}(1), v_{f}(2)$ be the number of vertices of $G$ having labels $0,1,2$ respectively under $f$ and $e_{f}(0), e_{f}(1), e_{f}(2)$ be the number of edges having labels $0,1,2$ respectively under $f^{*}$.

DEFINITION 5. A ternary vertex labeling of a graph $G$ is called a 3-equitable labeling if $\left|v_{f}(i)-v_{f}(j)\right| \leq 1$ and $\left|e_{f}(i)-e_{f}(j)\right| \leq 1$ for all $0 \leq i, j \leq 2$. A graph $G$ is 3 equitable if it admits 3-equitable labeling.

The concept of 3-equitable labeling was introduced by Cahit [8]. Many researchers have studied 3-equitability of graphs. Cahit [8] proved that $C_{n}$ is 3 -equitable except $n \equiv 3(\bmod 6)$. In the same paper he proved that an Eulerian graph with number of edges congruent to 3 (mod6) is not 3-equitable. Youssef [9] proved that $W_{n}$ is 3 -equitable for all $n \geq 4$.

DEFINITION 6. A prime cordial labeling of a graph $G$ with vertex set $V(G)$ is a bijection $f: V(G) \rightarrow\{1,2,3, \ldots,|V|\}$ 
defined by

$$
\begin{aligned}
f(e=u v) & =1 & & \text { ifgcd }(f(u), f(v))=1 \\
& =0 & & \text { otherwise }
\end{aligned}
$$

and $\left|e_{f}(0)-e_{f}(1)\right| \leq 1$. A graph which admits prime cordial labeling is called a prime cordial graph.

The concept of prime cordial labeling was introduced by Sundaram et al.[10] and in the same paper they investigate several results on prime cordial labeling. Vaidya and Vihol [11] have also discussed prime cordial labeling in the context of graph operations.

By combining the concepts of prime cordial labeling and 3equitable labeling and by using the result in Number Theory that if $\operatorname{gcd}(a, b)=1$, then $\operatorname{gcd}(a+b, a-b)=1$ or 2 , we introduce a new concept called 3-equitable prime cordial labeling as follows.

DEFINITION 7. A 3-equitable prime cordial labeling of a graph $G$ with vertex set $V(G)$ is a bijection $f: V(G) \rightarrow$ $\{1,2,3, \ldots, p\}$ defined by

$f(e=u v)=\left\{\begin{array}{l}1 \quad \text { if } \operatorname{gcd}(f(u), f(v))=1 \\ \text { and } \operatorname{gcd}(f(u)+f(v), f(u)-f(v))=1 \\ 2 \quad \text { if } \operatorname{gcd}(f(u), f(v))=1 \\ \quad \text { and } \operatorname{gcd}(f(u)+f(v), f(u)-f(v))=2 \\ 0 \quad \text { otherwise }\end{array}\right.$

and $\left|e_{f}(i)-e_{f}(j)\right| \leq 1$ for all $0 \leq i, j \leq 2$. A graph which admits 3-equitable prime cordial labeling is called a 3-equitable prime cordial graph.

\section{MAIN RESULTS}

Here we prove that paths $P_{n}$, cycles $C_{n}, n \geq 4$, stars $K_{1, n}, n \equiv 1(\bmod 3)$ and complete graphs $K_{n}, n \leq 2$ admit 3 -equitable prime cordial labeling.

\section{THEOREM 1. The path $P_{n}$ is 3-equitable prime cordial.}

Proof. Let $P_{n}: u_{1} u_{2} \ldots u_{n}$ be the path and let $t_{i}=$ $u_{i} u_{i+1}(1 \leq \mathrm{i} \leq \mathrm{n}-1)$ be the edges.

Case 1: $n \leq 4$

The following table gives the 3 -equitable prime cordial labeling of $P_{n}, n \leq 4$.

Vertex labels:

\begin{tabular}{|c|c|c|c|c|}
\hline$n$ & $u_{1}$ & $u_{2}$ & $u_{3}$ & $u_{4}$ \\
\hline 1 & 1 & & & \\
\hline 2 & 2 & 1 & & \\
\hline 3 & 2 & 1 & 3 & \\
\hline 4 & 2 & 4 & 1 & 3 \\
\hline
\end{tabular}

Case 2: $n \geq 5$

Let $v_{k}$ be the $k^{t h}$ vertex in $P_{n-1}$.

Subcase $1: n \equiv 1,2(\bmod 3)$

Define

$$
\begin{aligned}
u_{k} & =v_{k} \quad \text { if } \quad k \leq n-1 \\
\text { and } u_{n} & =n
\end{aligned}
$$

Subcase $2: n \equiv 0(\bmod 3)$

Define

$$
\begin{aligned}
u_{k} & =v_{k} \quad \text { if } \quad k \leq n-2 \\
u_{n-1} & =n \\
\text { and } u_{n} & =n-1
\end{aligned}
$$

Thus in both cases we have $\left|e_{f}(i)-e_{f}(j)\right| \leq 1$ for all $0 \leq$ $i, j \leq 2$.

Hence $P_{n}$ is 3 -equitable prime cordial
EXAMPLE 1. The 3-equitable prime cordial labeling of paths $P_{n}(5 \leq n \leq 13)$ with vertex labels and the corresponding edge labels are given in the following tables.

Vertex labels:

\begin{tabular}{|l|l|l|l|l|l|l|l|l|l|l|l|l|l|}
\hline$n$ & $u_{1}$ & $u_{2}$ & $u_{3}$ & $u_{4}$ & $u_{5}$ & $u_{6}$ & $u_{7}$ & $u_{8}$ & $u_{9}$ & $u_{10}$ & $u_{11}$ & $u_{12}$ & $u_{13}$ \\
\hline 5 & 2 & 4 & 1 & 3 & 5 & & & & & & & & \\
\hline 6 & 2 & 4 & 1 & 3 & 6 & 5 & & & & & & & \\
\hline 7 & 2 & 4 & 1 & 3 & 6 & 5 & 7 & & & & & & \\
\hline 8 & 2 & 4 & 1 & 3 & 6 & 5 & 7 & 8 & & & & & \\
\hline 9 & 2 & 4 & 1 & 3 & 6 & 5 & 7 & 9 & 8 & & & & \\
\hline 10 & 2 & 4 & 1 & 3 & 6 & 5 & 7 & 9 & 8 & 10 & & & \\
\hline 11 & 2 & 4 & 1 & 3 & 6 & 5 & 7 & 9 & 8 & 10 & 11 & & \\
\hline 12 & 2 & 4 & 1 & 3 & 6 & 5 & 7 & 9 & 8 & 10 & 12 & 11 & \\
\hline 13 & 2 & 4 & 1 & 3 & 6 & 5 & 7 & 9 & 8 & 10 & 12 & 11 & 13 \\
\hline
\end{tabular}

Edge labels:

\begin{tabular}{|l|l|l|l|l|l|l|l|l|l|l|l|l|}
\hline$n$ & $t_{1}$ & $t_{2}$ & $t_{3}$ & $t_{4}$ & $t_{5}$ & $t_{6}$ & $t_{7}$ & $t_{8}$ & $t_{9}$ & $t_{10}$ & $t_{11}$ & $t_{12}$ \\
\hline 2 & 1 & & & & & & & & & & & \\
\hline 3 & 1 & 2 & & & & & & & & & & \\
\hline 4 & 0 & 1 & 2 & & & & & & & & & \\
\hline 5 & 0 & 1 & 2 & 2 & & & & & & & & \\
\hline 6 & 0 & 1 & 2 & 0 & 1 & & & & & & & \\
\hline 7 & 0 & 1 & 2 & 0 & 1 & 2 & & & & & & \\
\hline 8 & 0 & 1 & 2 & 0 & 1 & 2 & 1 & & & & & \\
\hline 9 & 0 & 1 & 2 & 0 & 1 & 2 & 2 & 1 & & & & \\
\hline 10 & 0 & 1 & 2 & 0 & 1 & 2 & 2 & 1 & 0 & & & \\
\hline 11 & 0 & 1 & 2 & 0 & 1 & 2 & 2 & 1 & 0 & 1 & & \\
\hline 12 & 0 & 1 & 2 & 0 & 1 & 2 & 2 & 1 & 0 & 0 & 1 & \\
\hline 13 & 0 & 1 & 2 & 0 & 1 & 2 & 2 & 1 & 0 & 0 & 1 & 2 \\
\hline
\end{tabular}

THEOREM 2. The cycle $C_{n}$ is 3-equitable prime cordial if and only if $n \geq 4$.

Proof. Let $C_{n}: u_{1} u_{2} \ldots u_{n} u_{1}$ be the cycle and let $t_{i}=$ $u_{i} u_{i+1}(1 \leq \mathrm{i} \leq \mathrm{n}-1)$ and $t_{n}=u_{n} u_{1}$ be the edges.

Case 1: $n=3$

The labeling of $C_{3}$ is given as follows.

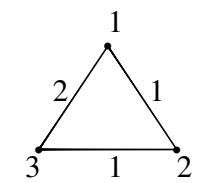

Fig 1: Labeling of $C_{3}$

We see that $\left|e_{f}(0)-e_{f}(1)\right|=2$ and hence $C_{3}$ is not 3 -equitable prime cordial.

Case 2: $4 \leq \mathrm{n} \leq 6$

The following table gives the 3-equitable prime cordial labeling of $C_{n}, 4 \leq \mathrm{n} \leq 6$.

Vertex labels:

\begin{tabular}{|c|c|c|c|c|c|c|}
\hline$n$ & $u_{1}$ & $u_{2}$ & $u_{3}$ & $u_{4}$ & $u_{5}$ & $u_{6}$ \\
\hline 4 & 3 & 2 & 4 & 1 & & \\
\hline 5 & 3 & 5 & 2 & 4 & 1 & \\
\hline 6 & 3 & 5 & 2 & 4 & 6 & 1 \\
\hline
\end{tabular}

Case 3: $n \geq 7$

Let $v_{k}$ be the $k^{t h}$ vertex in $C_{n-1}$.

Subcase $1: n \equiv 1,4,5(\bmod 6)$

Define

$$
\begin{aligned}
u_{k} & =v_{k} \quad \text { if } \quad k \leq n-1 \\
\text { and } u_{n} & =n
\end{aligned}
$$

Subcase $2: n \equiv 2(\bmod 6)$

Let $n=6 m+2$ 
Define

$$
\begin{aligned}
u_{k} & =v_{k} \quad \text { if } \quad k \leq 2 m \\
u_{2 m+1} & =v_{n-2} \\
u_{2 m+2} & =v_{n-1} \\
u_{k} & =v_{k-2} \quad \text { if } \quad 2 m+3 \leq k \leq n-1 \\
\text { and } u_{n} & =n
\end{aligned}
$$

Subcase $3: n \equiv 3(\bmod 6)$

Define

$$
\begin{aligned}
u_{k} & =v_{k} \quad \text { if } \quad k \leq n-2 \\
u_{n-1} & =n \\
\text { and } u_{n} & =n-1
\end{aligned}
$$

Subcase $4: n \equiv 0(\bmod 6)$

Define

$$
\begin{aligned}
u_{k} & =v_{k} \quad \text { if } \quad k \leq n-5 \\
u_{k} & =k \quad \text { if } \quad n-4 \leq k \leq n-2 \\
u_{n-1} & =n \\
\text { and } u_{n} & =n-1
\end{aligned}
$$

Thus in cases(ii)and(iii) we have $\left|e_{f}(i)-e_{f}(j)\right| \leq 1$ for all $0 \leq i, j \leq 2$.

Hence $C_{n}$ is 3-equitable prime cordial if and only if $n \geq 4$.

EXAMPLE 2. The 3-equitable prime cordial labeling of cycles $C_{n}(7 \leq n \leq 13)$ with vertex labels and the corresponding edge labels are given in the following tables.

Vertex labels:

\begin{tabular}{|l|c|c|c|c|c|c|c|c|c|c|c|c|c|}
\hline$n$ & $u_{1}$ & $u_{2}$ & $u_{3}$ & $u_{4}$ & $u_{5}$ & $u_{6}$ & $u_{7}$ & $u_{8}$ & $u_{9}$ & $u_{10}$ & $u_{11}$ & $u_{1}$ & $u_{1}$ \\
\hline 7 & 3 & 5 & 2 & 4 & 6 & 1 & 7 & & & & & & \\
\hline 8 & 3 & 5 & 1 & 7 & 2 & 4 & 6 & 8 & & & & & \\
\hline 9 & 3 & 5 & 1 & 7 & 2 & 4 & 6 & 9 & 8 & & & & \\
\hline 10 & 3 & 5 & 1 & 7 & 2 & 4 & 6 & 9 & 8 & 10 & & & \\
\hline 11 & 3 & 5 & 1 & 7 & 2 & 4 & 6 & 9 & 8 & 10 & 11 & & \\
\hline 12 & 3 & 5 & 1 & 7 & 2 & 4 & 6 & 8 & 9 & 10 & 12 & 11 & \\
\hline 13 & 3 & 5 & 1 & 7 & 2 & 4 & 6 & 8 & 9 & 10 & 12 & 11 & 13 \\
\hline
\end{tabular}

Edge labels:

\begin{tabular}{|c|c|c|c|c|c|c|c|c|c|c|c|c|c|}
\hline$n$ & $t_{1}$ & $t_{2}$ & $t_{3}$ & $t_{4}$ & $t_{5}$ & $t_{6}$ & $t_{7}$ & $t_{8}$ & $t_{9}$ & $t_{10}$ & $t_{11}$ & $t_{12}$ & $t_{13}$ \\
\hline 4 & 1 & 0 & 1 & 2 & & & & & & & & & \\
\hline 5 & 2 & 1 & 0 & 1 & 2 & & & & & & & & \\
\hline 6 & 2 & 1 & 0 & 0 & 1 & 2 & & & & & & & \\
\hline 7 & 2 & 1 & 0 & 0 & 1 & 2 & 2 & & & & & & \\
\hline 8 & 2 & 2 & 2 & 1 & 0 & 0 & 0 & 1 & & & & & \\
\hline 9 & 2 & 2 & 2 & 1 & 0 & 0 & 0 & 1 & 1 & & & & \\
\hline 10 & 2 & 2 & 2 & 1 & 0 & 0 & 0 & 1 & 0 & 1 & & & \\
\hline 11 & 2 & 2 & 2 & 1 & 0 & 0 & 0 & 1 & 0 & 1 & 2 & & \\
\hline 12 & 2 & 2 & 2 & 1 & 0 & 0 & 0 & 1 & 1 & 0 & 1 & 2 & \\
\hline 13 & 2 & 2 & 2 & 1 & 0 & 0 & 0 & 1 & 1 & 0 & 1 & 2 & 2 \\
\hline
\end{tabular}

THEOREM 3. The star graph $K_{1, n}$ is 3-equitable prime cordial if and only if $n \equiv 2(\bmod 3)$.

PROOF. Let $v$ be the central vertex and let $v_{1}, v_{2}, \ldots, v_{n}$ be the end vertices of the star $K_{1, n}$.

Case $1: n \equiv 2(\bmod 3)$

Assign the label 3 to the vertex $v$ and the remaining labels to the vertices $v_{1}, v_{2}, \ldots, v_{n}$.

We see that $\left|e_{f}(i)-e_{f}(j)\right| \leq 1$ for all $0 \leq i, j \leq 2$.

Thus $K_{1, n}$ is 3 -equitable prime cordial.
Case $2: n \equiv 0,1(\bmod 3)$

Subcase 1: Assign an even label to the vertex $v$.

There is no edge labeled 2 .

Subcase 2: Assign an odd label to the vertex $v$.

We see that $\left|e_{f}(1)-e_{f}(2)\right|=0$ or 1 but $\left|e_{f}(0)-e_{f}(1)\right| \geq 2$ and $\left|e_{f}(0)-e_{f}(2)\right| \geq 2$.

Thus $K_{1, n}$ is not 3-equitable prime cordial.

Hence $K_{1, n}$ is 3-equitable prime cordial if and only if $n \equiv$ $2(\bmod 3)$.

EXAMPLE 3. The 3-equitable prime cordial labeling of $K_{1,5}$ and $K_{1,8}$ are shown below.

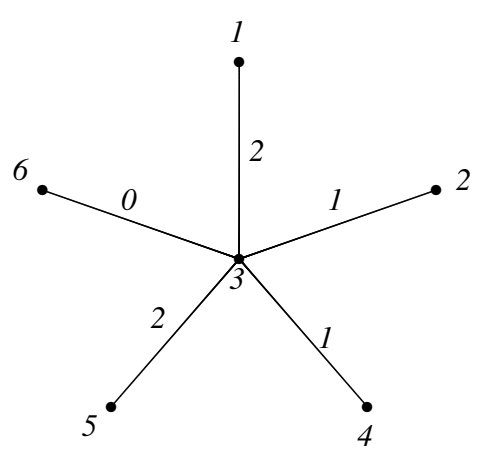

Fig 2: 3-equitable prime cordial labeling of $K_{1,5}$

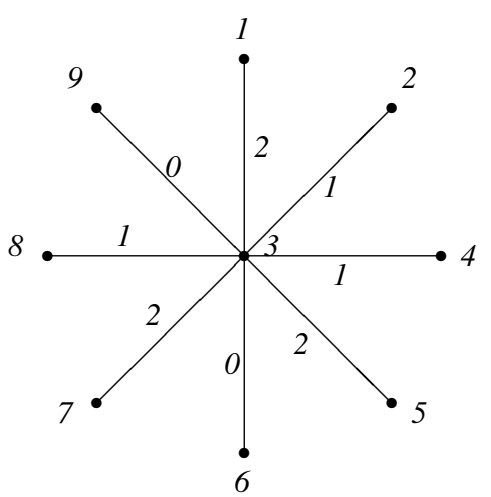

Fig 3: 3-equitable prime cordial labeling of $K_{1,8}$

THEOREM 4. The complete graph $K_{n}$ is not 3-equitable prime cordial for $n \geq 3$.

Proof. Let $v_{1}, v_{2}, \ldots, v_{n}$ be the vertices of $K_{n}$ with $f\left(v_{i}\right)=$ $i$.

Case(i): $n=1,2$

The 3-equitable prime cordial labeling of $K_{1}$ and $K_{2}$ are given as follows.

$$
\text { i } \quad \text { i } \quad \begin{array}{ll}
1 &
\end{array}
$$

Fig 4: 3-equitable prime cordial labeling of $K_{1}$ and $K_{2}$

Case(ii): $n=3$

By Fig 1 , we see that $\left|e_{f}(0)-e_{f}(1)\right|=2$ and hence $C_{3}$ is not 3 -equitable prime cordial.

Case(iii): $n \geq 4$

We see that $\left|e_{f}(0)-e_{f}(1)\right| \geq 3$ and hence $C_{3}$ is not 3-equitable prime cordial.

Thus $K_{n}$ is not 3-equitable prime cordial for $n \geq 3$. 


\section{CONCLUDING REMARKS}

By using a property from number theory, we have introduced 3 equitable prime cordial labeling of graphs. In the present work, we have tried to investigate the 3-equitable prime cordial labeling behaviour of standard graphs only. To investigate analogous results for different graphs as well as in the context of various graph labeling problems is an open area of research.

\section{ACKNOWLEDGEMENT}

The authors would like to thank Dr.K.Nagarajan, Associate Professor, Department of Mathematics, Sri S.R.N.M.College, Sattur-626 203, Tamil Nadu, India and Dr.S.Ilamaran, Associate Professor, Department of Mathematics, C.B.M.College, Coimbatore, Tamil Nadu, India for helping in the preparation of this article.

\section{REFERENCES}

[1] F. Harary, Graph Theory, Addition-Wesley, Reading, Mass, 1972.

[2] David M. Burton, Elementary Number Theory, Second Edition,Wm. C. Brown Company Publishers, 1980.
[3] J. A. Gallian, A dynamic survey of graph labeling, Electronic Journal of Combinatorics, 17 (2010), DS6.

[4] L.W.Beineke and S.M.Hegde, Strongly multiplicative graphs, Discuss.Math.Graph Theory, 21(2001), 63-75.

[5] G.S.Bloom and S.W.Golomb, Applications of numbered undirected graphs, Proceedings of IEEE, 165(4)(1977), 562-570.

[6] I.Cahit, Cordial graphs, A weaker version of graceful and harmonius graphs, Ars Combinatoria, 23(1987), 201-207.

[7] S.K.Vaidya, G.V.Ghodasara, Sweta Srivastav and V.J.Kaneria, Some new cordial graphs, Int.J.of Math and Math.Sci 4(2)(2008)81-92.

[8] I.Cahit, On cordial and 3-equitable labeling of graphs, Utilitas Math, 37(1990), 189-198.

[9] M.Z.Youssef, A necessary condition on k-equitable labelings, Utilitas Math, 64(2003), 193-195.

[10] M.Sundaram, R.Ponraj and S.Somasundaram, Prime cordial labeling of graphs, Journal of Indian Academy of Mathematics, 27(2005), 373-390.

[11] S.K.Vaidya and P.L.Vihol, Prime cordial labeling of some graphs, Modern Applied Science, 4(8)(2010), 119-126. 